\title{
APPLICATION OF DYNAMIC MAGNETIC FIELDS TO B16-BL6 MELANOMA CELLS LINKED WITH DECREASE IN CELLULAR VIABILITY AFTER SHORT EXPOSURES.
}

\author{
Lucas W.E. Tessaro ${ }^{\mathbf{1}}$, Lukasz M. Karbowski ${ }^{2}$, Robert M. Lafrenie ${ }^{\mathbf{3}}$ and Michael A. Persinger $^{\mathbf{4}}$ \\ *1,2,3,4 Behavioural Neuroscience Program, ${ }^{1,2,3,4}$ Department of Biology, ${ }^{2,3,4}$ Biomolecular Science Program ${ }^{3}$, \\ ${ }^{3}$ Laurentian University, Regional Cancer Program, \\ ${ }_{1,2,3,4}$ Sudbury Regional Hospital, Sudbury, On-tario, P3E 2 C6
}

*Corresponding Author: -

Email: lx_tessaro@laurentian.ca,Tel: 01-705-675-4824; Fax:01-705-671-3884.

\begin{abstract}
: -
Purpose: This experiment was designed to test the effects of an extremely low frequency electromagnetic field (ELF-EMF) generator ('Resonator') compared with strong static magnetic fields on cellular viability, and to illustrate the importance of dynamic patterns of fields compared with static patterns.

Materials and methods: B16-BL6 melanoma cells were exposed to ELF-EMF intensities ranging from 100-250 $\mu$ T or a strong static magnetic field (5000 G) for $3 \mathrm{~h}$. Following exposure cells were stained with trypan blue and observed under light microscopy. Manual cell counts were then conducted to determine the number of living vs. dead cells.

Results: Cellular viability as a function of magnetic field exposure was significantly different, with a statistically smaller number of cells remaining viable after exposure to ELF-EMF than the static magnetic field, which showed no difference from controls.

Conclusions: These results indicate the importance of the spatial-temporal component of magnetic field exposures, demonstrating that static magnetic fields which by definition lack dynamic variability do not influence a dynamic system such as a cell.
\end{abstract}

Keywords: - rotating magnetic fields; electromagnetic fields; B16-BL6 melanoma; vibration; interleukins

\section{(c) $(\$)$}




\section{INTRODUCTION}

Investigations into bio-electromagnetism have been conducted since the $19^{\text {th }}$ century (Santini, Rainaldi et al. 2009), with initial forays by Galvani, Récamier and Pravaz showing a method of destroying cancer cells through the use of electricity (Barnothy 1964, Stavroulakis 2003). As studies developed to investigate the magnetic component of electrical applications, it was first theorized that the mechanism of action of electromagnetic fields (EMFs) was through an increase in heat in tissues, but it has now been shown that the biological effects of EMFs are attributed influence within cellular communication through a number of mechanisms (Barnothy 1964, Adey 1981, Adey 1993, Stavroulakis 2003). Applications of low intensity, non-thermal EMFs are now known to have numerous physiological effects, many beneficial and therapeutic in various clinical settings (Pilla 2003).

These mechanisms by which magnetic fields are able to induce their effects on cellular systems remain somewhat contentious. For example, neonatal rats exposed to a $50 \mathrm{~Hz}$ sinusoidal EMF of $3 \mu \mathrm{T}$ for $4 \mathrm{~h} /$ day were shown to have significant morphological pathologies within their myocardium as opposed to control animals, attributed to oxidative stress and induced apoptosis (Tayefi, Kiray et al. 2010, Kiray, Tayefi et al. 2013). A similar study has shown that exposed for 14 days at $3 \mathrm{~h}$ per day to a $50 \mathrm{~Hz}$ sinusoidal EMF of $2.5 \mathrm{G}$ exposure led to an abnormal distribution of proteins within skeletal muscle (Söker, Sert et al. 2011). The varied biological effects of EMFs reported over the years are not without contrary evidence, such as the study by Söker and colleagues noting no discernable effects within myocardial tissue exposed to EMFs (Söker, Sert et al. 2011).

A number of studies have suggested that DNA itself may be the target of EMFs, for example a $60 \mathrm{~Hz}, 0.2-0.3 \mu \mathrm{T}$ field to promoted $\mathrm{Na}, \mathrm{K}-\mathrm{ATPase}$, with biosynthesis and transcription at a threshold of $0.8 \mu \mathrm{T}$ (Blank and Goodman 1997, Blank and Goodman 1999, Goodman and Blank 2002). Furthermore, a number of studies have suggested that the specific genomic target of EMFs are heat-shock proteins (hsp), particularly hsp70 which shows increased expression after exposure to a $60 \mathrm{~Hz}$ sinusoidal pattern of $8 \mu \mathrm{T}$ (Goodman, Blank et al. 1994, Goodman and Blank 1998, Kwee, Raskmark et al. 2001, Lim, Cook et al. 2005). Consequently, the argument can be made that the induction of cellular systems into EMFs is a form of stress, although this remains debatable (Lin, Opler et al. 1997).

Additional biological effects have been reported in the planarian flatworm, Dugesia tigrina, where it was reported that whole-body exposures to $3.8 \mu \mathrm{T}$ patterned EMFs led to increased rates of regeneration largely through cellular pathways similar to those outlined above (Tessaro and Persinger 2013); or five consecutive days of $6.5 \mathrm{~h}$ exposure to a frequency modulated magnetic field $(0.1$ to $2 \mu \mathrm{T})$, immediately followed by an additional

$6.5 \mathrm{~h}$ exposure on the fifth day to another complex field $(0.5$ to $5 \mu \mathrm{T})$ led to complete cellular disintegration (Murugan, Karbowski et al. 2013).

Work conducted by Buckner (Buckner 2011) on B16-BL6 melanoma cells has clearly demonstrated that exposure to a weak, temporally patterned EMF, known as 'Thomas' (W Thomas, Kavaliers et al. 1997) can significantly alter the level of intracellular $\mathrm{Ca}^{2+}$, as demonstrated by an increase in green fluorescence compared with controls. Once removed from the field, however, proliferation continues as though the fields were never applied. The cancer inhibiting effects of this field were also confirmed by $\mathrm{Hu}$ et al. (Hu, St-Pierre et al. 2010) in addition to the analgesic effects of the 'Thomas' pulse (Martin, Koren et al. 2004).

In the present study, B16-BL6 melanoma cancer cells were utilized in the test paradigm due to their propensity for being highly aggressive tumours. Given the high rates of vascularization and their invasiveness, even treatment with chemotherapy and surgery leads to a low survival rate (Lang 2002). The discovery of any alternative treatment would therefore be highly advantageous to diagnoses. We present results which indicate that in vitro single exposures to a dynamic-static magnetic field system, termed the 'Resonator', can significantly lower the viability counts of B16melanoma cells.

\section{Materials and Methods \\ Cell Cultures and Counting Techniques}

B16-BL6 mouse melanoma cell cultures were incubated in 150x20 mm tissue culture plates (Sarstedt, Laval, Qc) in Dulbecco's Modified Essential Medium (DMEM, Hyclone, Logan, UT) supplemented with 10\% fetal bovine serum, 100 $\mu \mathrm{g} / \mathrm{mL}$ streptomycin, and $100 \mathrm{U} / \mathrm{mL}$ penicillin (Invitrogen, Burlington, ON). The cells were in $37^{\circ} \mathrm{C}$ and $5 \% \mathrm{CO}_{2}$ and grown to approximately $75 \%$ confluence for the purpose of these experiments. Whole plates were taken directly from the incubator and placed within the experimental conditions for a duration of $3 \mathrm{~h}$ at room temperature $\left(25^{\circ} \mathrm{C}\right)$ including controls.

After exposure, the cell monolayers were harvested using $0.25 \%$ trypsin-EDTA and transferred into $1.5 \mathrm{~mL}$ Eppendorf tubes, which were then inoculated with $10 \mu \mathrm{L}$ of trypan blue stain and left to incubate at room temperature for 5 min. 10 $\mu \mathrm{L}$ samples were then loaded onto a hemocytometer and manual cell counts of white and blue cells were conducted under light microscopy. Eight counts per condition were completed for each of 16 experimental trials.

\section{Magnetic Field Exposures}

In total, three types of magnetic field exposure were utilized in this experiment. The first involved placing the whole culture plate directly on top of a strong, static magnetic 'puck' magnet. These 'pucks' have an average magnetic field intensity of $5000 \mathrm{G}$. The magnet is a composite of 12-ovoid neodymium magnets $(4 \mathrm{~cm} \mathrm{x} 1 \mathrm{~cm})$ set in a circular plastic casing $(9.5 \mathrm{~cm}$ x $5 \mathrm{~cm})$ in a 'Tree of Life' formation (Figure 1), designed and supplied by Dr. Kim Shallcross. 


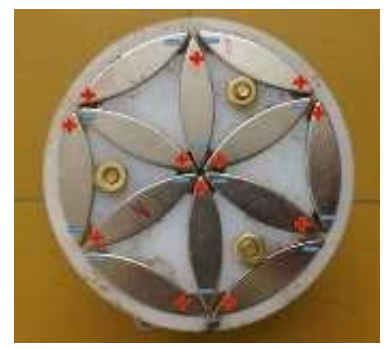

Figure 1: The puck magnets utilized in this experiment

The second magnetic field condition involved utilizing two of these 'puck' magnets and attaching them to two identical Graham Ball/Disc Variable Speed Transmission motors (Graham Transmission, Inc., Menomonee Falls, Wis.). Using the transmission motors the 'pucks' were rotated at approximately $1 \mathrm{~Hz}$ in opposite directions. Cell cultures were placed directly in the center of the rotating 'puck' magnetic fields.

The third magnetic field condition was created using the 'Resonator'. The 'Resonator' is comprised of set of six cylindrical neodymium magnets $(500 \mathrm{G})$ which are set into three axles. An electric motor rotated the three axles at equal speed ( 2000 rpms) by using a drive belt (Figure 2$)$. The entire device is encased in a wooden sound-dampening box (92 $\mathrm{cm} \times 92 \mathrm{~cm}$ x $155 \mathrm{~cm})$. The overall summed magnetic field was measured at $250 \mu \mathrm{T}$.

The precise schematics of the 'Resonator' are protected as Intellectual Property of Dr. Shallcross.

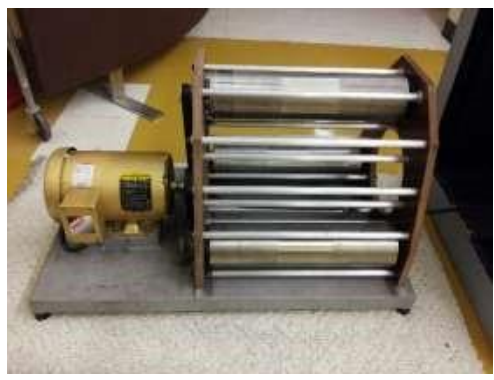

Figure 2: The 'Resonator' device with the soundproof cover removed. Shown is the motor drive system and motor belt (left) which rotates the three larger cylinders containing the magnets (right).

Figure 3 is a spectral density of the 'Resonator' magnetic field output recorded at $250 \mathrm{~Hz}$, taken above, along the wide side, and along its longitudinal axis. The primary frequencies associated with the field regardless of orthogonal location are $45 \mathrm{~Hz}, 88 \mathrm{~Hz}$, and $93 \mathrm{~Hz}$. The waveforms at these locations are shown in Figure 4.

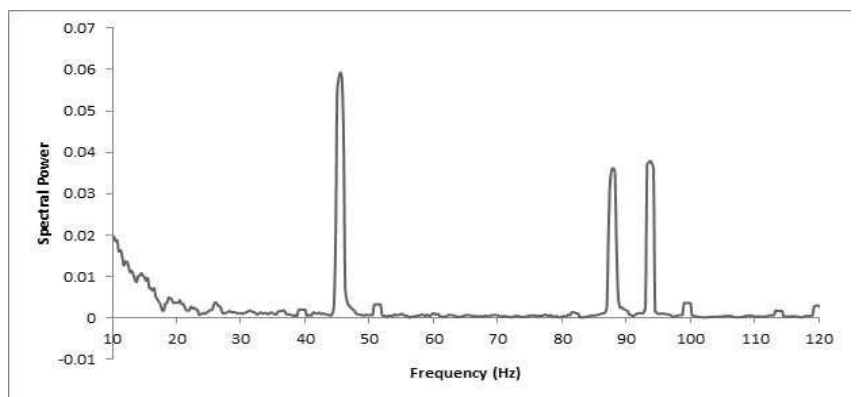

Figure 3: Spectral power density of the 'Resonator' magnetic field showing peaks at 45, 88 and $90 \mathrm{~Hz}$.

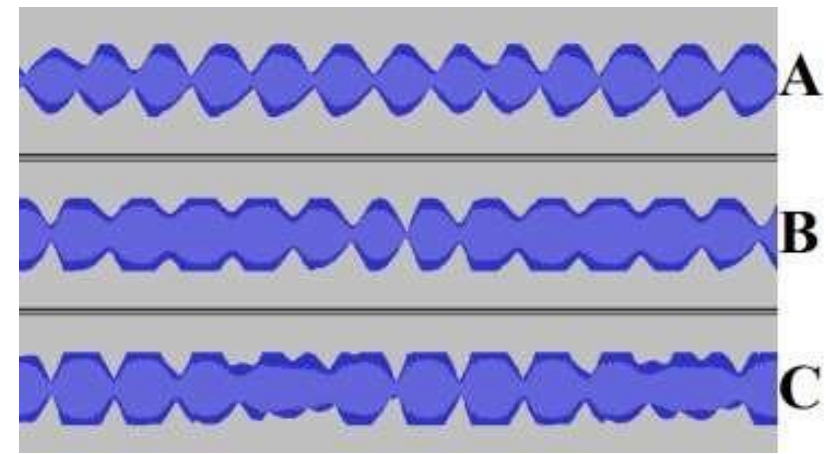

Figure 4: Waveforms of the magnetic field recorded from above the device (A), along the wide axis (B) and from directly in front of the device $(C)$.

When the device is off, the 'Resonator' has a static magnetic field. As illustrated in figure 5, the static DC field intensity of the surface of the device is quite variable. When the device is on, the field produced is $250 \mu \mathrm{T}$ when proximal to the 
upper surface. This intensity changes with respect to proximity: the cell cultures placed at $0 \mathrm{~m}$ measured an intensity of $250 \mu \mathrm{T}, 0.1 \mathrm{~m}$ at an intensity of $200 \mu \mathrm{T}$, and $1.0 \mathrm{~m}$ with an intensity of $100 \mu \mathrm{T}$ from the 'Resonator' for the duration of the exposure. No change in temperature of the room or within the vicinity of the 'Resonator' was noted for the duration of exposure measured using a digital thermometer.

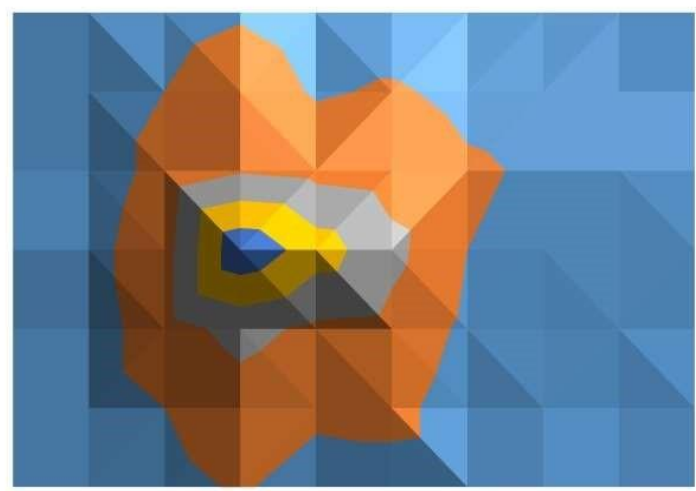

$$
\begin{aligned}
& =4-5 \\
& =3-4 \\
& =2-3 \\
& =1-2 \\
& =0-1
\end{aligned}
$$

Figure 5: Static field intensity map of the surface of the 'Resonator' when the device is turned off. Legend measurements in Gauss (G).

Due to the mechanical nature of the device, a vibrational component was noted within the vicinity of the 'Resonator'. Precise measurements of mechanical acceleration, velocity and displacement were recorded using an Extech Instruments 4-Channel Vibration Datalogger Model VB500 at $1 \mathrm{sec}$ intervals for $2 \mathrm{~min}$.

Statistical Analyses

A number of oneway analyses of variance and analyses of covariance were computed as a function of cell viability, calculated viability $=\left(\frac{\text { white cell count }}{\text { total cell count }}\right) \times 100 \%$. Post-hoc tests included combinations of Tukey's.

All analyses were completed with IBM Statistical Product and Service Solutions (SPSS) software.

Spectral density analyses were conducted using magnetic field intensities measured using an AC Milligauss Meter Model UHS (Alpha Labs, Inc., Salt Lake City, USA) which input directly to a computer using Audacity multi-track audio editor and recorder for Windows, with a $\Delta \mathrm{t}$ of $250 \mathrm{~Hz}$. Recordings in Audacity were exported into a text file which was then loaded into SPSS for spectral density analyses.

\section{Results}

A one-way analysis of variance of the viability counts by the type of magnetic field exposure revealed a significant condition effect $\left[\mathrm{F}_{(5,503)}=82.656, \mathrm{p}<.001, \eta^{2}=0.45\right]$. Post-hoc analyses indicated that the three 'Resonator' conditions had significantly lower percentage of viable cells (more mortality) as compared to controls and the two 'puck' magnet conditions, which did not differ from one another or controls. On average, the 'Resonator' viability counts were $\sim 75 \%$ compared with non-'Resonator' averages of $\sim 90 \%$, suggesting a reduction in viability of $\sim 15 \%$ (Figure 6 ).

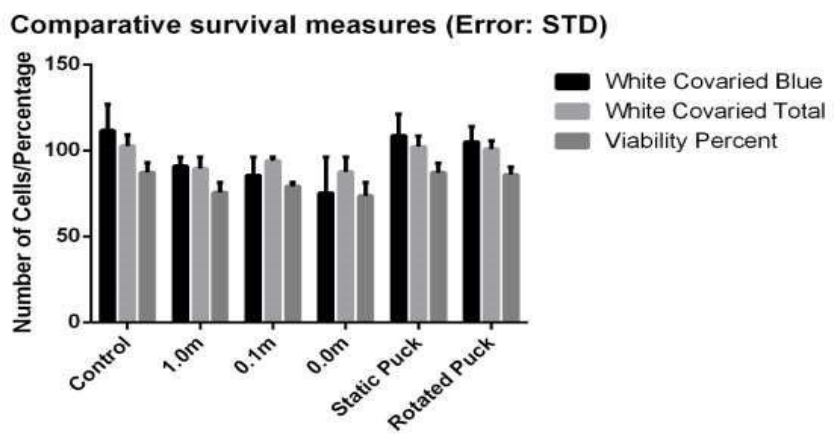

Figure 6: Results of the 3h exposures of BL6-B16 melanoma cancer cells to the 'Resonator', static and rotating puck magnets compared with control (no field) condition.

A further exploration of these data compared the number of white (living) cells with the number of blue (dead) cells. In this analysis, there was again a significant difference between the six magnetic field conditions for the numbers of white cells when co-varied for the number of blue cells $\left[\mathrm{F}_{(5,503)}=5.310, \mathrm{p}<.001, \eta^{2}=0.049\right]$. Subsequent post-hoc tests corroborated the previous analyses that the 'Resonator' conditions were the major source of the significant differences. Similar results were noted when the co-variate was the total number of cells counted $\left[\mathrm{F}_{(5,503)}=54.778, \mathrm{p}<.001, \eta^{2}=0.015\right]$. Z-score values of the data were calculated and graphed in Figure 7. It is apparent that the 'Resonator' conditions increased the relative number of blue cell counts which coincided with the reported lower viability counts of the entire condition, and to a lesser extent the number of white cells. Additionally, the positive and negative Z-scores were also significantly 
deviated from chance, as indicated by the dispersion measures. This also means that compared with controls and the 'puck' conditions, the 'Resonator' conditions showed a significant increase in the measures (i.e. white counts, blue counts, and viability counts) equivalent to the difference of one standard error measurement.

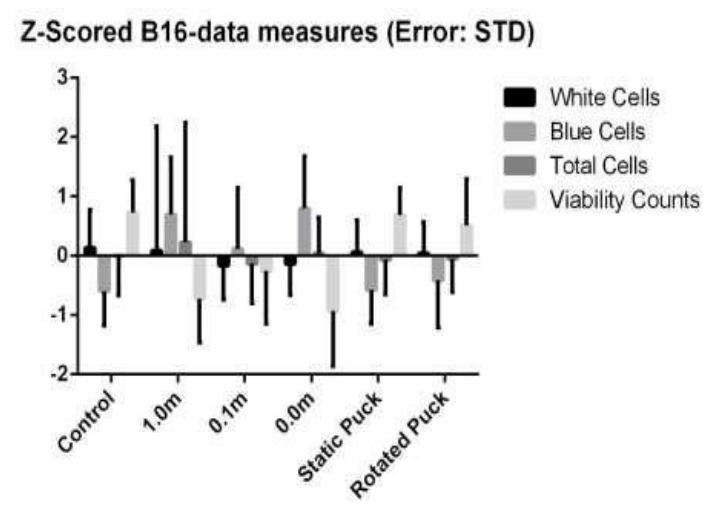

Figure 7: Z-scored values of all experimental measures. The figure illustrates that the blue cell counts increase with respect to the reported lower viability counts.

\section{Discussion}

The results of our experiment using a static $0.5 \mathrm{~T}$ static magnetic field elicited no significant difference in cellular viability, which does not support the results of a previous study using $7 \mathrm{~T}$ static magnetic fields which arrested the proliferation of melanoma cells by $20 \%$ in vitro (Raylman, Clavo et al. 1996). The intensities of these fields differ by a full magnitude which could explain the lack of significance in the present study.

In more recent years the beneficial application of magnetic fields as a cancer therapy have been presented in literature. Yamaguchi et al. (Yamaguchi, Ogiue-Ikeda et al. 2006) utilized a pulsed magnetic field application in the range of 2500 $\mathrm{G}$ which reduced the size of melanoma tumors in vivo. Work conducted by $\mathrm{Hu}$ et al. (Hu, St-Pierre et al. 2010) utilized fields which were lower than those used in the present experiment $(0.003 \mu \mathrm{T}, 3.5 \mu \mathrm{T})$ but for an identical duration $(3 \mathrm{~h})$ with results inhibiting the growth of in vivo tumors. The magnetic field patterns were quite dissimilar, which suggests the pattern of the magnetic field rather than raw power (i.e. intensities) is the critical feature.

The results of this study also press the importance of the spatial-temporal component of magnetic field applications. Despite having higher intensities, the 'puck' magnet conditions elicited no discernable effects. The importance of the spatial-temporal patterns of magnetic fields to generate biological effects was demonstrated by Buckner (Buckner, 2011). Furthermore, a number of reviews of the biological effects of magnetic fields have hinted at the necessity for both physiologically patterned and spatial-temporal variability as a component for biological interactions (Adey, 1981; Santini, Rainaldi et al., 2009).

The construction of the 'Resonator' itself can be inferred as being similar to an electromagnetic field. The rotation of the magnets at regular intervals generates a defined temporal pattern (Figures 3 and 4). The question remains as to the possible mechanism by which the 'Resonator' induces its effect. Yamaguchi et al. (Yamaguchi, Ogiue-Ikeda et al. 2006) outlined the role for interleukin-2 (IL-2) and tissue-necrosis-factor- $\alpha$ (TNF- $\alpha$ ) as possible mechanisms, as both were up-regulated after exposure to a $0.25 \mathrm{~T}, 25 \mathrm{~Hz}$ EMF. The former is involved with immunosuppression (Nelson, 2004) while the latter is associated with tumor-suppression (Aggarwal, 2003). Magnetic field exposures are also frequently associated with $\mathrm{Ca}^{2+}$ intake as well as the generation of reactive-oxygen species (Shahidain, 2001; Nelson, 2004).

There was a perceivable vibration component to the magnetic field exposure, with the displacement recorded between 4 $20 \mu \mathrm{m}$, dependant on distance from the 'Resonator'. This is within the average width of a cell. It is interesting to note that previous works have suggested that vibration exposures in the duration of $3 \mathrm{~h}$ can affect a number of cellular processes and signalling cascades, most notably the interleukin pathways (Curry, Bain et al. 2002, Russo, Banes et al. 2002, Puig, Rico et al. 2005, Nowak, Pawlak et al. 2012).

When the mechanical acceleration of the 'Resonator' at $0 \mathrm{~m}\left(4.5 \mathrm{~m} / \mathrm{s}^{2}\right)$ is applied to the average mass of a cell including water weight $\left(5.2 \times 10^{-13} \mathrm{~kg}\right)$ the resultant force is $1.48 \times 10^{-12} \mathrm{~N}$. What is quite interesting is that when this force is applied across the width of the cellular membrane (average $10^{-8} \mathrm{~m}$ ), the resultant energy is $2.24 \times 10^{-20} \mathrm{~J}$. This value has been demonstrated to effectively be a neuromolecular quantum of energy, being involved with the maintenance of the cellular membrane resting potential, cell-adhesion forces, and cellular metabolism (Persinger, 2010). This would lend credence to the argument that the vibrational forces recorded play a definitive role in the effects of the 'Resonator'.

This same calculation can be repeated for the $0.1 \mathrm{~m}$ and $1.0 \mathrm{~m}$ recordings of vibrational acceleration, in which case the resultant energies are $7.33 \times 10^{-21} \mathrm{~J}$ and $1.56 \times 10^{-21} \mathrm{~J}$. At first this may suggest that these are energies are incapable of affecting membrane potentials, it is a known feature of cancer cells that their membrane potentials are in the order of -10 to $-30 \mathrm{mV}$ (Levin 2013). If the quanta of energy is calculated for a required depolarization of a cancer cell membrane, the value is $3.24 \times 10^{-21} \mathrm{~J}$ - well within the range of the 'Resonator' induced energies.

In fact, the mechanism may not be mechanical at all. In a recent review article, Persinger (Persinger, 2014) noted the vastly underrepresented effects of infrasonic forces. Given the 'Resonator' frequency peaks are roughly $45 \mathrm{and} 90 \mathrm{~Hz}$, well within the infrasonic range, the mechanism may not be electromagnetic or mechanical (vibrational) in nature. The frequency of the magnetic field across the surface area of the cell at $0.1 \mathrm{~m}$ for the $45 \mathrm{~Hz}$ peak is equal to the frequency of the $90 \mathrm{~Hz}$ peak at $1.0 \mathrm{~m}-689 \mathrm{~Hz}$ - suggesting an innate feature within the device to, quite literally, resonate within cells. 
A similarly conspicuous circumstantial calculation is noted with the Lorentz force of the magnetic field is calculated and converted to an equivalent frequency $-82 \mathrm{~Hz}$, within the range of the secondary 'Resonator' peak.

The 'Resonator' is a very unique device and its operation remains somewhat mysterious. Physical measurements of its mechanical action and electromagnetic field are a step towards understanding its full range of effects on local biological systems. The interrelatedness of infrasound and vibrational interactions suggest that in this particular case the mechanism of action is an aggregate of EMF and classical mechanics.

\section{Declaration of Interest}

The authors report no declarations of interest.

\section{References}

[1].Adey, W. R. (1981). "Tissue interactions with nonionizing electromagnetic fields." Physiological reviews $61(2): 435$.

[2].Adey, W. R. (1993). "Biological effects of electromagnetic fields." Journal of cellular biochemistry 51: $410-410$.

[3].Aggarwal, B. B. (2003). "Signalling pathways of the TNF superfamily: a double-edged sword." Nature Reviews Immunology 3(9): 745-756.

[4].Barnothy, M. F. (1964). Biological effects of magnetic fields, Plenum Press New York.

[5].Blank, M. and R. Goodman (1997). "Do electromagnetic fields interact directly with DNA?" Bioelectromagnetics 18(2): 111-115.

[6].Blank, M. and R. Goodman (1999). "Electromagnetic fields may act directly on DNA." Journal of cellular biochemistry 75(3): 369-374.

[7].Buckner, C. A. (2011). Effects of electromagnetic fields on biological processes are spatial and temporaldependent. $\mathrm{PhD}$, Laurentian University.

[8].Curry, B. D., J. L. Bain, J. G. Yan, L. L. Zhang, M. Yamaguchi, H. S. Matloub and D. A. Riley (2002). "Vibration injury damages arterial endothelial cells." Muscle \& nerve 25(4): 527-534.

[9].Goodman, R. and M. Blank (1998). "Magnetic field stress induces expression of hsp70." Cell stress \& chaperones 3(2): 79 .

[10]. Goodman, R. and M. Blank (2002). "Insights into electromagnetic interaction mechanisms." Journal of cellular physiology 192(1): 16-22.

[11]. Goodman, R., M. Blank, H. Lin, R. Dai, O. Khorkova, L. Soo, D. Weisbrot and A. Henderson (1994). "Increased levels of hsp70 transcripts induced when cells are exposed to low frequency electromagnetic fields." Bioelectrochemistry and bioenergetics 33(2): 115-120.

[12]. Hu, J. H., L. S. St-Pierre, C. A. Buckner, R. M. Lafrenie and M. A. Persinger (2010). "Growth of injected melanoma cells is suppressed by whole body exposure to specific spatial-temporal configurations of weak intensity magnetic fields." International journal of radiation biology 86(2): 79-88.

[13]. Kiray, A., H. Tayefi, M. Kiray, H. A. Bagriyanik, C. Pekcetin, B. U. Ergur and C. Ozogul (2013). "The effects of exposure to electromagnetic field on rat myocardium." Toxicology and Industrial Health 29(5): 418-425.

[14]. Kwee, S., P. Raskmark and S. Velizarov (2001). "Changes in cellular proteins due to environmental non-ionizing radiation. I. Heat-shock proteins." Electromagnetic Biology and Medicine 20(2): 141-152.

[15]. Lang, P. G. (2002). "Current concepts in the management of patients with melanoma." American journal of clinical dermatology 3(6): 401-426.

[16]. Levin, M. (2013). "Reprogramming cells and tissue patterning via bioelectrical pathways: molecular mechanisms and biomedical opportunities." Wiley Interdisciplinary Reviews: Systems Biology and Medicine 5(6): 657-676.

[17]. Lim, H. B., G. G. Cook, A. T. Barker and L. A. Coulton (2005). "Effect of $900 \mathrm{MHz}$ electromagnetic fields on nonthermal induction of heat-shock proteins in human leukocytes." Radiation research 163(1): 45-52.

[18]. Lin, H., M. Opler, M. Head, M. Blank and R. Goodman (1997). "Electromagnetic field exposure induces rapid, transitory heat shock factor activation in human cells." Journal of cellular biochemistry 66(4): 482-488.

[19]. Martin, L., S. Koren and M. Persinger (2004). "Thermal analgesic effects from weak, complex magnetic fields and pharmacological interactions." Pharmacology Biochemistry and Behavior 78(2): 217-227.

[20]. Murugan, N. J., L. M. Karbowski, R. M. Lafrenie and M. A. Persinger (2013). "Temporally-Patterned Magnetic Fields Induce Complete Fragmentation in Planaria." PloS one 8(4): e61714.

[21]. Nelson, B. H. (2004). "IL-2, regulatory T cells, and tolerance." The Journal of Immunology 172(7): 3983-3988. Nowak, A., M. Pawlak, M. Brychcy, J. Celichowski and P. Krutki (2012). "Effects of brief whole-body vibration on bone metabolic and immunological indices in rats."

[22]. Persinger, M. (2010). "10-20 Joules as a Neuromolecular Quantum in Medicinal Chemistry: An Alternative Approach to Myriad Molecular Pathways?" Current Medicinal Chemistry 17(27): 3094-3098.

[23]. Persinger, M. A. (2014). "Infrasound, human health, and adaptation: an integrative overview of recondite hazards in a complex environment." Natural Hazards 70(1): 501-525.

[24]. Pilla, A. A. (2003). "Weak time-varying and static magnetic fields: from mechanisms to therapeutic applications." Biological Effects of Electromagnetic Fields, Stavroulakis P, ed., Springer Verlag: 34-75.

[25]. Puig, F., F. Rico, I. Almendros, J. M. Montserrat, D. Navajas and R. Farre (2005). "Vibration enhances interleukin8 release in a cell model of snoring induced airway inflammation." SLEEP-NEW YORK THEN WESTCHESTER28(10): 1312. 
[26]. Raylman, R. R., A. C. Clavo and R. L. Wahl (1996). "Exposure to strong static magnetic field slows the growth of human cancer cells in vitro."

[27]. Russo, A., A. Banes, M. Elfervig and M. Tsuzaki (2002). The effect of vibration on annulus cell signaling. Proceedings of the NASS 16th Annual Meeting/The Spine Journal, Elsevier.

[28]. Santini, M. T., G. Rainaldi and P. L. Indovina (2009). "Cellular effects of extremely low frequency (ELF) electromagnetic fields." International Journal of Radiation Biology 85(4): 294-313.

[29]. Shahidain, R. M., JE Sisken, R (2001). "Calcium spiking activity and baseline calcium levels in ROS 17/2.8 cells exposed to extremely low frequency electromagnetic fields (ELF EMF)." International journal of radiation biology 77(2): 241-248.

[30]. Söker, S., C. Sert, M. Deniz, I. Tekmen, M. Akkus, Y. Nergiz, S. SÖKER, C. SERT, M. DENIZ and I. TEKMEN (2011). "The Effects of Electromagnetic Fields on the Ultrastructure of Heart." Int. J. Morphol 29(3): 960-964.

[31]. Stavroulakis, P. (2003). Biological effects of electromagnetic fields: Mechanisms, modeling, biological effects, therapeutic effects, international standards, exposure criteria, Springer.

[32]. Tayefi, H., A. Kiray, M. Kiray, B. U. Ergur, H. A. Bagriyanik, C. Pekcetin, M. Fidan and C. Ozogul (2010). "The effects of prenatal and neonatal exposure to electromagnetic fields on infant rat myocardium." Arch Med Sci 6(6): 837-842.

[33]. Tessaro, L. W. and M. A. Persinger (2013). "Optimal durations of single exposures to a frequency-modulated magnetic fieldimmediately after bisection in planarian predict final growth values." Bioelectromagnetics.

[34]. W Thomas, A., M. Kavaliers, F. S. Prato and K.-P. Ossenkopp (1997). "Antinociceptive effects of a pulsed magnetic field in the land snail, $<\mathrm{i}>$ Cepaea nemoralis $</ \mathrm{i}>$." Neuroscience letters 222(2): 107-110.

[35]. Yamaguchi, S., M. Ogiue-Ikeda, M. Sekino and S. Ueno (2006). "Effects of pulsed magnetic stimulation on tumor development and immune functions in mice." Bioelectromagnetics 27(1): 64-72. 